\title{
Supercritical and transcritical real-fluid mixing using the PC-SAFT EOS
}

\author{
C. Rodriguez ${ }^{\star 1}$, A. Vidal ${ }^{1}$, P. Koukouvinis ${ }^{1}$, M. Gavaises ${ }^{1}$ \\ ${ }^{1}$ School of Mathematics, Computer Science and Engineering, City, University of London, UK \\ *Corresponding author: Carlos.Rodriguez@city.ac.uk
}

\begin{abstract}
A numerical framework has been developed to simulate the mixing of supercritical and transcritical fluids using an equation of state based on statistical associating fluid theory. In a Diesel engine the liquid fuel is injected into supercritical air. After the injection, the Diesel is heated over its critical temperature reaching a supercritical state. Modelling real-fluid effects is critical in order to properly characterize the air/fuel mixing in the combustion chamber. By using the PC-SAFT EoS (Perturbed Chain Statistical Association Fluid Theory Equation of State) real fluids effects can be taken into account in a CFD simulation. The PC-SAFT EoS shows best results than cubic EoS computing liquid density, compressibility, speed of sound, vapor pressures and density derivatives. Unlike cubic EoS, this model accounts for the shape and size of the molecules. Gas, liquid, supercritical and vapor-liquid equilibrium states can be simulated. PT FLASH (Isothermal Multiphase Flash Calculation) is applied to compute the phase diagram used by the code. Shock tube problems were conducted in a wide range of pressures and densities using $n$-dodecane to show the capability of the developed algorithm. The results were compared with the solution of an exact Riemann solver which has the PC-SAFT EoS implemented showing a high degree of agreement. In addition, a two-dimensional simulation of supercritical nitrogen jet mixing was carried out to check the multidimensional capability of the code.
\end{abstract}

\section{Keywords}

Supercritical, transcritical, PC-SAFT EoS

\section{Introduction}

Small soot particles generated in Diesel engines are linked to important health issues such as heart attacks, premature death, bronchitis or asthma among children. Recent studies [1] has shown that a supercritical Diesel combustion can reduce the emissions of particulate matter and nitrogen oxides. The significant reduction (or even almost disappearance) of the fuel vaporization time has been linked with reduced harmful emissions [2, 3] while at the same time, it may improve engine thermodynamic efficiency due to engine operation at higher compression ratios.

In Diesel engines the liquid fuel is injected into air that has pressure and temperature conditions higher than the critical point of the fuel. The liquid injection temperature is lower than the fuel critical temperature but as the liquid is heated up, it may reach supercritical temperature before its full vaporisation. This process is known as transcritical injection. A single fluid or a mixture reaches a supercritical state when the pressure and the temperature exceed its critical values. Under such conditions, the repulsive atomic forces overcome the surface tension, resulting in the existence of a single phase. A supercritical phase shows properties of both gases and liquids (e.g., gas-like diffusivity and liquid-like density).

Simulating supercritical flows makes necessary the implementation of a real-gas equation of state (EoS), which takes into account the intermolecular repulsive forces in the relationship among pressure, density and temperature. Nowadays, cubic EoS are standard to compute supercritical and transcritical thermodynamics in CFD simulations. Soave-Redlich-Kwong and Peng-Robinson EoS are widely utilised. By using the Péneloux volume correction [4], the low values of liquid density provided by the original version of these models can be fixed, but the calculation of gas compressibility factors and pressure derivatives are still inaccurate [5].

In the last few decades more advanced models have been developed. The SAFT EoS is an advanced molecular model which can precisely compute the thermodynamic properties of complex mixtures. It is based on the perturbation theory extensively studied by Wertheim [6-9]. Chapman et al. [10,11] developed this EoS by applying Wertheim's theory and extending it to mixtures. 
In the SAFT model the reference fluid is formed by spherical segments of equal size. Then attractive forces are added between molecules. After this, chains are forms with hard-sphere segments and finally associative forces are added between molecules. Figure 1 shows a physical representation of the different contributions.
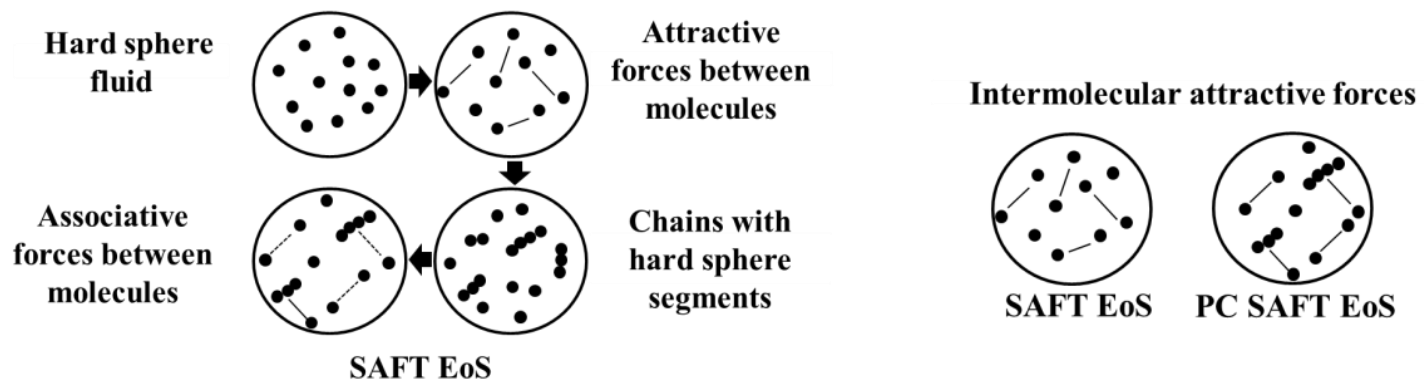

Figure 1. Physical description of the attractive and repulsive contributions of the SAFT EoS and the PC-SAFT EoS [12]

Many variants of the SAFT model exist. Among them, the PC-SAFT EoS (Perturbed Chain Statistical Association Fluid Theory EoS) is the one implemented here. In this variant, hard chains are used as the reference fluid instead of hard spheres. The SAFT EoS compute the attractive interaction between individual molecules and chain segments while in the PC-SAFT EoS the interactions between entire molecules are considered. This improves the thermodynamic description of chain-like fluid mixtures [12].

Although this EoS is based on advance molecular theory, only three parameters of each component have to be specified to compute the thermodynamic properties of the mixtures. The performance of the PC-SAFT model is superior to cubic EoS computing heat capacities, speed of sound, pressure-volume derivative and pressuretemperature derivative [13]. The results for gas Z-factors and compressibility are more accurate as well. It shows a good prediction of liquid densities and pure component vapor pressures. Additionally, it accounts for the shape and size of the molecules as it is based on statistical mechanics.

\section{Mathematical formulation}

The Navier-Stokes equations for a non-reacting multi-component mixture containing $\mathrm{N}$ species in a $2 \mathrm{D}$ flow are given by:

$\frac{\partial \mathbf{U}}{\partial \mathrm{t}}+\frac{\partial \mathbf{F}}{\partial \mathrm{x}}+\frac{\partial \mathbf{G}}{\partial \mathrm{y}}=\frac{\partial \mathbf{F}_{\mathrm{v}}}{\partial \mathrm{x}}+\frac{\partial \mathbf{G}_{\mathrm{v}}}{\partial \mathrm{y}}$

The vectors of eq. 1 are:

$\mathbf{U}=\left[\begin{array}{c}\rho \mathrm{Y}_{1} \\ \vdots \\ \rho \mathrm{Y}_{\mathrm{N}} \\ \rho u \\ \rho \mathrm{v} \\ \rho E\end{array}\right], \mathbf{F}=\left[\begin{array}{c}\rho u Y_{1} \\ \vdots \\ \rho u Y_{N} \\ \rho u^{2}+p \\ \rho u v \\ (\rho E+p) u\end{array}\right], \mathbf{G}=\left[\begin{array}{c}\rho u Y_{1} \\ \vdots \\ \rho u Y_{N} \\ \rho u v \\ \rho \mathrm{v}^{2}+p \\ (\rho E+p) v\end{array}\right], \mathbf{F}_{\mathrm{v}}=\left[\begin{array}{c}-\mathrm{J}_{\mathrm{x}, 1} \\ \vdots \\ -\mathrm{J}_{\mathrm{x}, \mathrm{N}} \\ \sigma_{\mathrm{xx}} \\ \sigma_{\mathrm{xy}} \\ \mathrm{u} \sigma_{\mathrm{xx}}+\mathrm{v} \sigma_{\mathrm{xy}}-\mathrm{q}_{\mathrm{x}}\end{array}\right]$

$\mathbf{G}_{\mathbf{v}}=\left[\begin{array}{c}-\mathbf{J}_{\mathrm{y}, 1} \\ \vdots \\ -\mathbf{J}_{\mathrm{y}, \mathrm{N}} \\ \sigma_{\mathrm{yx}} \\ \sigma_{\mathrm{yy}} \\ \mathrm{u} \sigma_{\mathrm{yx}}+\mathrm{v} \sigma_{\mathrm{yy}}-\mathrm{q}_{\mathrm{y}}\end{array}\right]$ 
where $\rho$ is the fluid density, $\mathrm{u}$ and $\mathrm{V}$ are the velocity components, $\mathrm{p}$ is the pressure, $\mathrm{E}$ is the total energy, $\mathrm{J}_{\mathrm{i}}$ is the mass diffusion flux of species $\mathrm{i}, \sigma$ is the deviatoric stress tensor and $\mathrm{q}$ is the diffusion heat flux vector.

A finite volume scheme is employed in this work. The PC-SAFT EoS is implemented in order to simulate supercritical and transcritical states. Operator splitting is adopted to divide the physical processes into hyperbolic and parabolic sub-steps. The global time step is computed using the CFL criterion of the hyperbolic operator.

\subsection{Hyperbolic sub-step}

The HLLC solver is used to solve the Riemann problem. The conservative variables are interpolated onto the cell faces using a fifth-order WENO (Weighted Essentially Non-Oscillatory) scheme due to its high order accuracy and non-oscillatory behaviour. Time integration is performed by using a third order TVD RK (Total Variation Diminishing Runge-Kutta) method.

\subsection{Parabolic sub-step}

The method developed by Chung et al. [14] is used to calculate the values of the dynamic viscosity and thermal conductivity of the mixture. In order to compute the diffusion coefficient the model of Riazi et al. [15] is utilised. A TVD scheme of third order is used to perform the time integration of this sub-step. The diffusion fluxes are computed conservatively using a second order method.

\subsection{PC-SAFT EOS}

In perturbation theory, the potential energy of the molecular fluid is described as the sum of the potential energy of the reference fluid and a perturbation. The PC-SAFT EOS (based on perturbation theory) is expressed as the sum of all the residual Helmholtz free energy contributions. These contributions correspond to the different types of molecular interactions. The Helmholtz free energy was computed using eq.3 [16]

$\tilde{a}^{r e s}=\tilde{a}^{h c}+\tilde{a}^{\text {disp }}$

$\tilde{a}^{h c}=\bar{m} \tilde{a}^{h s}-\sum_{i} x_{i}\left(m_{i}-1\right) \ln g_{i i}^{h s}\left(\sigma_{d i i}\right)$

$\tilde{a}^{h s}=\frac{1}{\varsigma_{0}}\left[\frac{3 \varsigma_{1} \varsigma_{2}}{\left(1-\varsigma_{3}\right)}+\frac{\varsigma_{2}^{3}}{\varsigma_{3}\left(1-\varsigma_{3}\right)^{2}}+\left(\frac{\varsigma_{2}^{3}}{\varsigma_{3}^{2}}-\varsigma_{0}\right) \ln \left(1-\varsigma_{3}\right)\right]$

$\tilde{a}^{\text {disp }}=-2 \pi \rho_{m} I_{1}(\eta, \bar{m}) \overline{m^{2} \varepsilon \sigma_{d}^{3}}-\pi \rho_{m} \bar{m} C_{1} I_{2}(\eta, \bar{m}) \overline{m^{2} \varepsilon^{2} \sigma_{d}^{3}}$

$$
\varsigma_{n}=\frac{\pi}{6} \rho_{m} \sum_{i} x_{1} m_{i} d_{i}^{n} \quad n \in\{0,1,2,3\}
$$

The thermodynamic variables computed by the PC-SAFT model are the temperature, pressure, sonic fluid velocity, enthalpy, entropy, fugacity and Gibbs free energy. The algorithm inputs are the density, internal energy and three pure component parameters (number of segments per chain, energy parameter of each component and segment diameter). The density and the internal energy are obtained from the conservative variables of the CFD code. The pure component parameters are specified in the initialization of the simulation.

A Newton Raphson method is employed to compute the temperature. The temperature is required to calculate the value of all other thermodynamic variables. The temperature dependent function used in the iterative method is the internal energy. When this converges to the value provided by the conservative variables, the iterative process is terminated.

\subsubsection{Iteration process}

a) Guessing an initial temperature value

The value of the temperature from the previous time RK sub-step or the previous time step is used to initialize the iteration process. In most cells this value will be close to the solution (assuming a small time step). 
b) Computing the compressibility factor

It is calculated as the sum of the ideal gas contribution (considered to be 1), the dispersion contribution and the residual hard-chain contribution [16].

$Z=1+Z^{h c}+Z^{\text {disp }}$

$Z^{h s}=\frac{\varsigma_{3}}{\left(1-\varsigma_{3}\right)}+\frac{3 \varsigma_{1} \varsigma_{2}}{\varsigma_{0}\left(1-\varsigma_{3}\right)^{2}}+\frac{3 \varsigma_{2}^{3}-\varsigma_{3} \varsigma_{2}^{3}}{\varsigma_{0}\left(1-\varsigma_{3}\right)^{3}}$

$Z^{h c}=\bar{m} Z^{h s}-\sum_{i} x_{i}\left(m_{i}-1\right)\left(g_{i i}^{h s}\right)^{-1} \rho_{m} \frac{\partial g_{i i}^{h s}}{\partial \rho_{m}}$

$Z^{d i s p}=-2 \pi \rho_{m} \frac{\partial\left(\eta I_{1}\right)}{\partial \eta} \overline{m^{2} \varepsilon \sigma_{d}^{3}}-\pi \rho_{m} \bar{m}\left[C_{1} \frac{\partial\left(\eta I_{2}\right)}{\partial \eta}+C_{2} \eta I_{2}\right] \overline{m^{2} \varepsilon^{2} \sigma_{d}^{3}}$

Where $\mathrm{C}_{1}, \mathrm{C}_{2}$ are abbreviations [16].

c) Computing the pressure

The pressure can be calculated using eq.12 once the compressibility factor is known [16].

$P=Z k T \rho_{m}\left(10^{10}\right)^{3}$

d) Computing the internal energy

It is the sum of the ideal internal energy and the residual internal energy. The residual internal energy is calculated by using eq.13 [17].

$\frac{U^{r e s}}{R T}=-T\left(\frac{\partial \tilde{a}^{r e s}}{\partial T}\right)_{\rho, x_{i}}$

If the computed total internal energy is not close enough to the value obtained from the conservative variables the Newton Raphson method is applied to calculate a new value of the temperature and repeat steps b-d.

\subsubsection{Other thermodynamic properties}

Once the temperature value is known the following properties are computed.

Heat capacities: They are computed as the sum of the ideal contribution (Poling et al. [18]) and the correction done by the PC-SAFT EoS (eq.15) [17].

$$
C_{v}^{r e s}=-R T\left[2\left(\frac{\partial \tilde{a}^{r e s}}{\partial T}\right)_{\rho, x_{i}}+T\left(\frac{\partial^{2} \tilde{a}^{r e s}}{\partial T^{2}}\right)_{\rho, x_{i}}\right], C_{p}^{r e s}=C_{v}^{r e s}+R \frac{\left[\rho_{m} T\left(\frac{\partial^{2} \tilde{a}^{r e s}}{\partial \rho_{m} \partial T}\right)_{x_{i}}+\rho_{m}\left(\frac{\partial \tilde{a}^{r e s}}{\partial \rho_{m}}\right)_{T, x_{i}}+1\right]^{2}}{\left[\rho_{m}{ }^{2}\left(\frac{\partial^{2} \tilde{a}^{r e s}}{\partial \rho_{m}{ }^{2}}\right)_{T, x_{i}}+2 \rho_{m}\left(\frac{\partial \tilde{a}^{r e s}}{\partial \rho_{m}}\right)_{T, x_{i}}+1\right]}
$$

Total enthalpy: It is used to compute the thermal diffusion vector in the parabolic sub-step. The total enthalpy is the sum of the ideal contribution (obtained by integrating the ideal heat capacity at constant pressure with respect to the temperature) and the residual enthalpy (eq.14) [16].

$\frac{\hat{h}^{r e s}}{R T}=-T\left(\frac{\partial \tilde{a}^{r e s}}{\partial T}\right)_{\rho, x_{i}}+(Z-1)$ 
Total entropy: Likewise the enthalpy, it is the sum of the ideal and the residual contributions. The ideal contribution is computed using the formula of the entropy for ideal gases and the residual contribution is computed using eq. 16 [16].

$$
\frac{\hat{s}^{r e s}(P, T)}{R}=-T\left[\left(\frac{\partial \tilde{a}^{r e s}}{\partial T}\right)_{\rho, x_{i}}+\frac{\tilde{a}^{r e s}}{T}\right]+\ln (Z)
$$

Sonic fluid velocity: The equation applied by Diamantonis et al. (eq.17) [19] was used.

$$
\omega=\sqrt{\frac{C_{p}}{C_{v}}\left(\frac{\partial P}{\partial \rho_{m}}\right)_{T}}
$$

Fugacity: It is an input of the PT FLASH model used to perform phase equilibrium calculations. Eq.18 [16] is applied to compute the fugacity.

$$
\ln \varphi_{k}=\frac{\mu_{k}^{r e s}(T, v)}{k T}-\ln Z
$$

\subsubsection{Calculation of the phase diagram}

The calculation of the number of phases present in a mixture in a certain condition is a recognized problem in the utilization of any EoS. In some cases, the number of phases is assumed a priori and then the composition in every phase is calculated by imposing the conditions of equilibrium. However, this technique often leads to divergence in the iterative methods used to achieve these. In our case, this is solved by an isothermal flash calculation after a stability analysis using the Tangent Plane Criterion Method proposed by Michelsen [20] and applied to the PCSAFT EoS by Justo-Garcia et al. [21]. Nevertheless, for a case in which we have a single component, this problem gets simplified vastly and the rudimentary method of assuming a fixed number of phases can be applied. First, we calculate the saturation curve for the single component by imposing the equilibrium conditions:

$$
\begin{aligned}
& T_{\text {SATURATED_VAPOR }}=T_{\text {SATURATED_LIQUID }} \\
& p_{\text {SATURATED_VAPOR }}=p_{\text {SATURATED_LIQUID }} \\
& \mu_{\text {SATURATED_VAPOR }}=\mu_{\text {SATURATED_LIQUID }}
\end{aligned}
$$

where $\mathrm{T}, \mathrm{p}$ and $\mu$ are the temperature, pressure and chemical potential of the component. Then for the inputs, we compare with the calculated saturation curve, knowing then if the conditions for the component are inside or outside of it. In case of being outside, the EoS is applied directly. On the other hand, if it is inside, the saturation values are weighted by the amount of vapor-liquid calculated. The phase diagram is computed in the initialization of the program in order to reduce the computational time of the simulation.

\section{Results and discussion}

In this section, the hyperbolic part of the code is validated against the exact solution of the Riemann problem. In addition, a two-dimensional simulation of a cryogenic nitrogen jet was performed to demonstrate the twodimensional capability of the numerical framework.

\subsection{Shock tube problem}

A shock tube problem is a one-dimensional Riemann problem frequently applied to validate computational fluid codes. The Euler equations are solved in this validation so a direct comparison with the exact solver can be done. The component used to perform the simulation was $n$-dodecane. The exact Riemann solver uses an unstructured table which contains the thermodynamic properties derived from the PC-SAFT EoS. This table is generated before performing the simulations. The domain used was $\mathrm{x} \in[-2,2]$, the initial conditions of the left state are $\rho_{L}=751.167 \mathrm{~kg} / \mathrm{m}^{3}, \quad p_{L}=40.2 \mathrm{kPa}, U_{L}=0 \mathrm{~m} / \mathrm{s}$ and the initial conditions of the right state are $\rho_{R}=719.249 \mathrm{~kg} / \mathrm{m}^{3}$, $p_{R}=170 \mathrm{bar}, u_{R}=0 \mathrm{~m} / \mathrm{s}$. 800 equally spaced cells were used with a fifth order spatial accuracy. Wave transmissive boundary conditions are implemented in the left and right sides. Figure 3 shows a high degree of agreement between the exact solution and the computed results. 

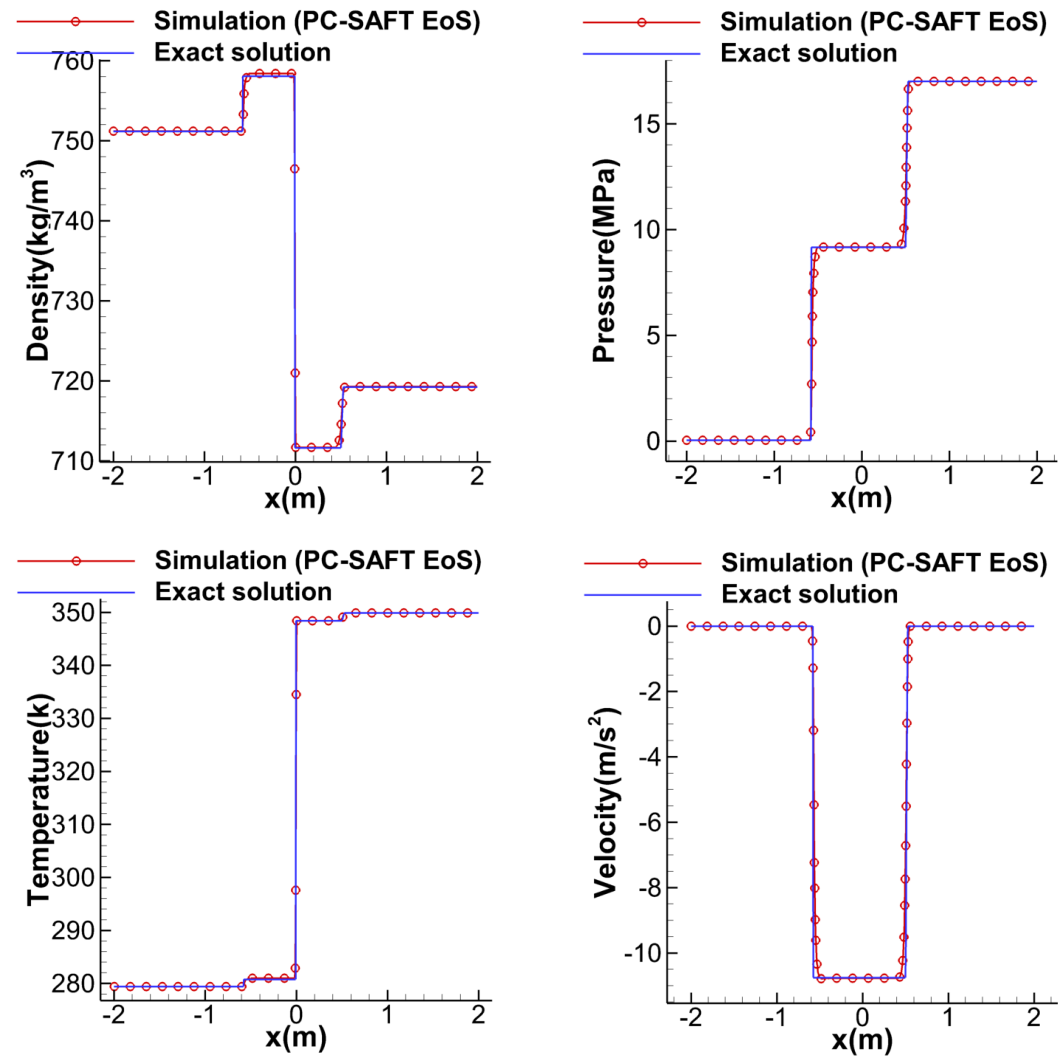

Figure 2. Validation of the hyperbolic sub-step in the Riemann problem. Comparison between the exact and the numerical solution of the density (upper left), pressure (upper right), temperature (lower left) and $\mathrm{x}$-velocity (lower right) at $\mathrm{t}=5 \times 10^{-4} \mathrm{~S}$.

\subsection{Two-dimensional test case}

A two-dimensional supercritical nitrogen jet mixing case was simulated using the developed numerical framework. Cryogenic liquid nitrogen is injected into a chamber filled with high temperature nitrogen. This simulation was previously done by Ma et al. [22] employing similar conditions to the case 3 of Mayer et al. [23]. The ambient pressure in the combustion chamber is $4 \mathrm{MPa}$, the density of the jet is $440 \mathrm{~kg} / \mathrm{m}^{3}$ and the density of the nitrogen in the combustion chamber is $40 \mathrm{~kg} / \mathrm{m}^{3}$. The velocity of the jet is $50 \mathrm{~m} / \mathrm{s}$ and the diameter of the exit nozzle is $1.0 \mathrm{~mm}$. The domain used is $30 \mathrm{~mm} \times 15 \mathrm{~mm}$. A structured mesh is applied with a uniform cell distribution. The cell size is $0.06 \mathrm{~mm} \times 0.06 \mathrm{~mm}$. A flat velocity profile is imposed at the inlet. Transmissive boundary conditions are used in all boundaries with the exception of the inlet. It was not utilised a sub-grid scale model. The parabolic sub-step is included into the simulation.

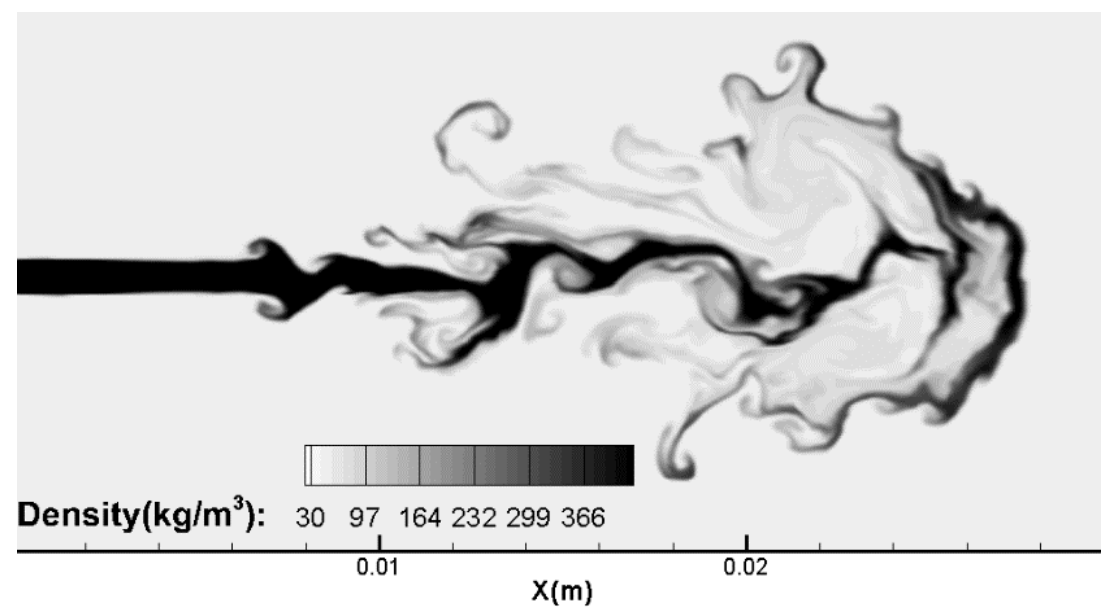

Figure 3. Density results of the simulation of the planar cryogenic nitrogen jet at $t=7.90 \times 10^{-4} \mathrm{~s}$ 
At high pressures the intermolecular interactions in the gas-liquid interphase are symmetrical so the atomization phenomena is suppress. In Figure 3 it can be appreciated that the mixing process depends on the turbulence where the large density gradients play an important role. When the jet is introduced in the high temperature environment of the chamber the velocity gradients at the jet surface generate a rollup which finally breakup into ligament-shaped structures. The Kelvin Helmholtz instability can be observed in the shear layer.

The mixing layer is similar to a gas/gas turbulent mixing case. This feature was observed by the experiments performed by Chehroudi el al. [24]. No droplets are formed at this conditions. Figure 4 shows how the jet is quickly heated to a gas-like supercritical state after the injection takes place.

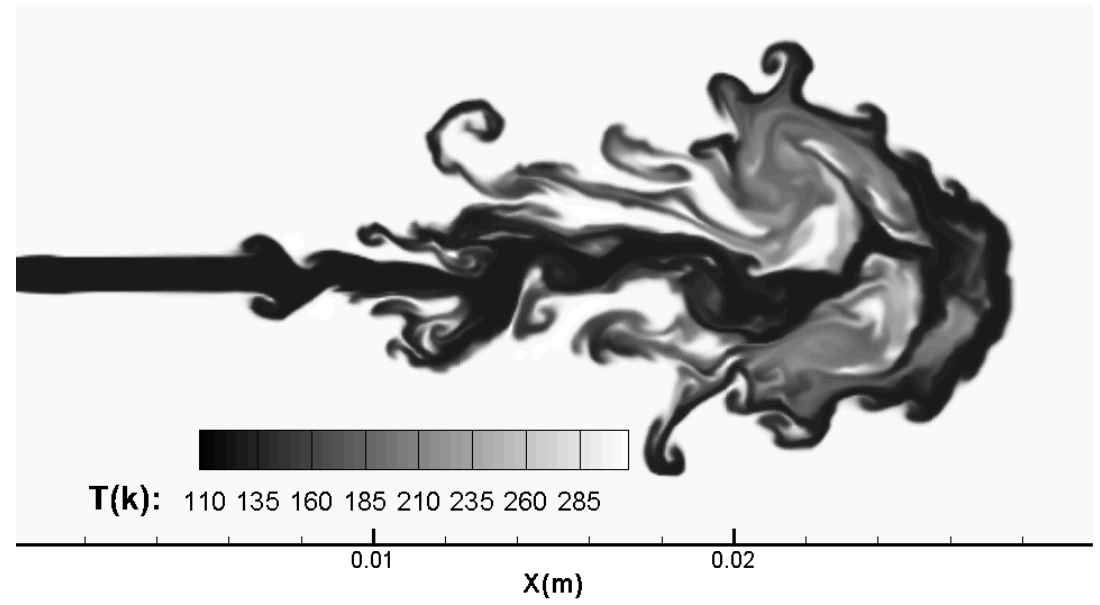

Figure 4. Temperature results of the simulation of the planar cryogenic nitrogen jet at $t=7.90 \times 10^{-4} \mathbf{S}$

By setting the CFL number at 1.0 and using a second order RK method the number of time steps needed to simulate $7.9 \mathrm{E}-4 \mathrm{~s}$ is approximately 50000 . The average CPU time for a time step is $14.2 \mathrm{~s}$. It has to be taken into account that this is a serial code used to validate the numerical framework and it is not optimized. If the operator splitting is not applied the CPU time can be reduced to 8.6s. Unlike the fifth order WENO scheme, the evaluated first and second order schemes are not able to properly capture the large density gradients. Third and fourth order reconstruction schemes have not been evaluated.

\section{Conclusions}

A numerical framework was developed to simulate the mixing of supercritical and transcritical flows using the PCSAFT EoS. By using this advance molecular model, real fluid effects are taken into account in the simulations. Apart from supercritical state, the developed code is able to simulate gas, liquid and vapor-liquid phase equilibrium. The algorithm applied to couple the PC-SAFT EoS and the Navier-Stokes equations allows a quick computation of the thermodynamic properties of the flow. The hyperbolic part of the code was validated against the exact solution of the Riemann problem solved for the PC-SAFT EoS. Furthermore, the simulation of the two-dimensional supercritical nitrogen jet mixing case shows the multidimensional capability of the numerical framework. Future work will include the simulation of transcritical and supercritical mixing cases with a composition more similar to Diesel in temperature and pressure conditions closer to those of a diesel engine.

\section{Acknowledgements}

This project has received funding from the European Union Horizon 2020 Research and Innovation programme. Grant Agreement No 675528

\section{Nomenclature}

$$
\begin{aligned}
& \text { List of Symbols } \\
& \qquad \begin{array}{cl}
\tilde{a}^{\text {res }} & =\text { reduced Helmholtz free energy } \\
d & =\text { temperature-dependent segment diameter }[\AA] \\
g & =\text { radial distribution function } \\
I & =\text { integrals of the perturbation theory }
\end{array}
\end{aligned}
$$

$$
\begin{aligned}
& \text { Greek } \text { Letters } \\
& \begin{array}{ll}
\varepsilon & =\text { depth of pair potential }[\mathrm{J}] \\
\eta & =\text { packing fraction } \\
\rho & =\text { density }\left[\mathrm{kg} / \mathrm{m}^{3}\right] \\
\rho_{m} & =\text { total number density of molecules }\left[1 / \AA^{3}\right]
\end{array}
\end{aligned}
$$




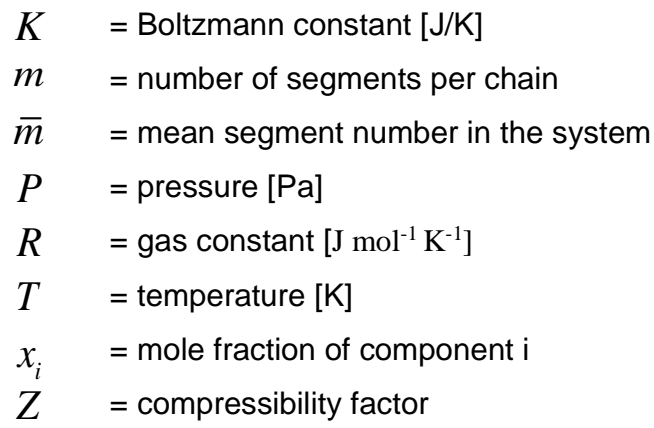

$\sigma_{d} \quad=$ segment diameter $\left[\AA^{3}\right]$

Superscripts

disp = contribution due to dispersive attraction

$h c=$ residual contribution of hard-chain system

$h s \quad=$ residual contribution of hard-sphere system

id = ideal gas contribution

\section{References}

[1] Lin, R. and Tavlarides, L.L., 2012. Thermophysical properties needed for the development of the supercritical diesel combustion technology: Evaluation of diesel fuel surrogate models. The Journal of Supercritical Fluids, 71, pp.136-146.

[2] Tavlarides, L.L. and Antiescu, G., Syracuse University, 2009. Supercritical diesel fuel composition, combustion process and fuel system. U.S. Patent 7,488,357.

[3] Vogel T. et. al, (2013). Transition of Fuel Components into Supercritical State under Diesel Process Conditions. In ILASS - Europe. Chania.

[4] Péneloux, A., Rauzy, E. and Fréze, R., 1982. A consistent correction for Redlich-Kwong-Soave volumes. Fluid Phase Equilibria, 8(1), pp.7-23.

[5] Schou Pedersen, K. and Hasdbjerg, C., 2007, January. PC-SAFT equation of state applied to petroleum reservoir fluids. In SPE Annual Technical Conference and Exhibition. Society of Petroleum Engineers.

[6] Wertheim, M.S., 1984. Fluids with highly directional attractive forces. I. Statistical thermodynamics. Journal of statistical physics, 35(1), pp.19-34.

[7] Wertheim, M.S., 1984. Fluids with highly directional attractive forces. II. Thermodynamic perturbation theory and integral equations. Journal of statistical physics, 35(1-2), pp.35-47.

[8] Wertheim, M.S., 1986. Fluids with highly directional attractive forces. III. Multiple attraction sites. Journal of statistical physics, 42(3), pp.459-476.

[9] Wertheim, M.S., 1986. Fluids with highly directional attractive forces. IV. Equilibrium polymerization. Journal of statistical physics, 42(3), pp.477-492.

[10] Chapman, W.G., Gubbins, K.E., Jackson, G. and Radosz, M., 1989. SAFT: equation-of-state solution model for associating fluids. Fluid Phase Equilibria, 52, pp.31-38.

[11] Chapman, W.G., Jackson, G. and Gubbins, K.E., 1988. Phase equilibria of associating fluids: chain molecules with multiple bonding sites. Molecular Physics, 65(5), pp.1057-1079.

[12] Khare, N.P., 2003. Predictive modeling of metal-catalyzed polyolefin processes.

[13] De Villiers, A.J., Schwarz, C.E., Burger, A.J. and Kontogeorgis, G.M., 2013. Evaluation of the PC-SAFT, SAFT and CPA equations of state in predicting derivative properties of selected non-polar and hydrogen-bonding compounds. Fluid Phase Equilibria, 338, pp.1-15.

[14] Chung, T.H., Ajlan, M., Lee, L.L. and Starling, K.E., 1988. Generalized multiparameter correlation for nonpolar and polar fluid transport properties. Ind. Eng. Chem. Res, 27(4), pp.671-679.

[15] Riazi, M.R. and Whitson, C.H., 1993. Estimating diffusion coefficients of dense fluids. Industrial and Engineering Chemistry Research, 32(12).

[16] Gross, J. and Sadowski, G., 2001. Perturbed-chain SAFT: An equation of state based on a perturbation theory for chain molecules. Industrial \& engineering chemistry research, 40(4), pp.1244-1260.

[17] Gord, M.F., Roozbahani, M., Rahbari, H.R. and Hosseini, S.J.H., 2013. Modeling thermodynamic properties of natural gas mixtures using perturbed-chain statistical associating fluid theory. Russian Journal of Applied Chemistry, 86(6), pp.867-878.

[18] Poling, B.E., Prausnitz, J.M. and O'connell, J.P., 2001. The properties of gases and liquids (Vol. 5). New York: Mcgraw-hill. Appendix A.

[19] Diamantonis, N.I. and Economou, I.G., 2011. Evaluation of SAFT and PC-SAFT EoS for the calculation of thermodynamic derivative properties of fluids related to carbon capture and sequestration.

[20] Michelsen, M.L., 1982. The isothermal flash problem. Part I. Stability. Fluid phase equilibria, 9(1), pp.1-19.

[21] Justo-García, D.N., García-Sánchez, F. and Romero-Martínez, A., 2008, March. Isothermal Multiphase Flash Calculations with the PC-SAFT Equation of State. In E. Díaz-Herrera and E. Juaristi eds.,, AIP Conference Proceedings (Vol. 979, No. 1, pp. 195-214). AIP.

[22] Ma, P.C., Bravo, L. and Ihme, M., 2015. Supercritical and transcritical real-fluid mixing in diesel engine applications (No. ARL-RP-0551). ARMY RESEARCH LAB ABERDEEN PROVING GROUND MD VEHICLE TECHNOLOGY DIRECTORATE.

[23] Mayer, W., Telaar, J., Branam, R., Schneider, G. and Hussong, J., 2003. Raman measurements of cryogenic injection at supercritical pressure. Heat and Mass Transfer, 39(8-9), pp.709-719.

[24] Chehroudi, B., Talley, D. and Coy, E., 2002. Visual characteristics and initial growth rates of round cryogenic jets at subcritical and supercritical pressures. Physics of Fluids, 14(2), pp.850-861. 